\title{
Innovación, un acercamiento a su concepto, alcance, elementos y capacidades que la generan
}

Innovation, an approach to its concept, reach, elements and capacities that generate it

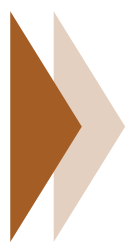

David Romero

Docente Facultad de Ciencias y Tecnologías - Universidad Santo Tomás. Ingeniero Industrial. Especialista en Gestión de Proyectos de Ingeniería. Candidato a magister en Política y Gestión de Ciencia y Tecnología.

E-mail: jesus.romero@ustadistancia.edu.co

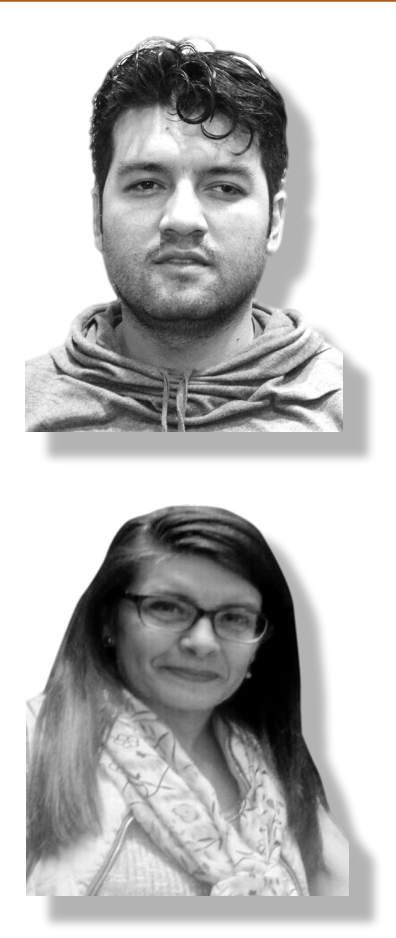

Flor Alba Pinzón

Docente Facultad de Ciencias y Tecnologías - Universidad Santo Tomás. Administradora de Empresas. Magister en Dirección Estratégica y Gerencia del Talento humano y MA. en Educación.

E-mail: floralbapinzon@ustadistancia.edu.co 


\section{RESUMEN}

La innovación es entendida como la forma de introducir algo a un producto, proceso, método de comercialización $u$ organizativo entre otros, "nuevo o significativamente mejorado" al mercado, por lo que es importante para los Países en Desarrollo (PED) determinar el alcance o diferentes niveles de la innovación, en un contexto determinado, y que para lograrlo se debe contar con un conjunto de capacidades (de absorción e innovación) y recursos, para así poder identificar las áreas en que se debe priorizar el fortalecimiento de la capacidad tecnológica.

Palabras Clave: alcance, contexto, capacidades tecnológicas, innovación, países en desarrollo.

\section{Abstract}

Innovation is understood as a way to introduce something to a product, process, marketing method or organizational among others, "new or significantly improved" the market, so it is important for Developing Countries (PED) determining the scope, different levels of innovation in a given context, and that to achieve this there must be a set of capabilities (absorption and innovation) and resources, in order to identify areas that should be prioritized strengthening technological capacity.

Keywords: Scope, context, technological capabilities, innovation, developing countries.
El presente artículo propone una discusión en torno a la pregunta ¿̇por qué es importante distinguir el alcance de la innovación? Partiendo del concepto de innovación y abordando a partir de los conceptos: capacidades tecnológicas, capacidades de innovación y capacidades de absorción, por qué para los Países en Desarrollo (PED) tendría sentido hacer más énfasis en el alcance de la innovación.

En primera instancia, es importante entender el concepto y alcance de la innovación, especialmente para los PED, porque permiten identificar las áreas en las que se debe priorizar el fortalecimiento de las capacidades tecnológicas que se requieren para la contribución de dicho desarrollo.

Se puede decir que la innovación es un fenómeno social ${ }^{1}$, que depende directamente de la aceptación y aprovechamiento que se tenga sobre una novedad (también puede darse capacidades tecnológicas, por la aparición de un invento ${ }^{4}$ ) al ser incorporado en un contexto determinado, que a su vez, surge como resultado del aprovechamiento o creación

1. Se utiliza el término fenómeno basado en la definición que da la Real Academia de la Lengua (RAE), así el fenómeno social descrito

representa una "manifestación que se hace presente a la consciencia de un sujeto y aparece como objeto de su percepción" (Real Academia

Española, 2014), en éste caso la innovación es la percepción dada por la sociedad o la interacción de sus actores, a la novedad introducida.

2. Una novedad no necesariamente es un invento; una novedad puede ser una nueva manera de utilizar algo ya existente, por otro lado un invento si representa una novedad al ser algo que no existía. El término novedad tiene mayor relación con un descubrimiento, que es el encontrar algo existente de lo cual no había registro alguno. 
de capacidades necesarias para la aplicación, transformacióny/ogeneración de conocimiento (tácito y explícito5), que permita lograr mejoras o solucionar problemas evidenciados, en la mayoría de las ocasiones y económicamente hablando, se considera que la innovación se da al introducir algo a un - producto, proceso, método de comercialización $\mathrm{u}$ organizativo entre otros - "nuevo o significativamente mejorado" al mercado (OEDC, 2005), es así como la condición de innovación está dada por la aceptación y los resultados en el uso o aplicación (aprovechamiento) de una novedad implementada en un contexto determinado.

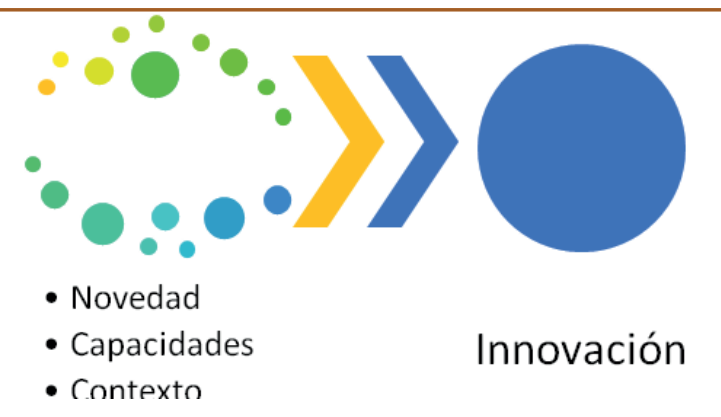

Figura 1. Elementos clave para la innovación.

Fuente. Elaboración propia.

Un ejemplo interesante de innovación es el caso de la empresa colombiana de productos alimenticios Ramo S.A. Nacida en 1950 como iniciativa del señor Rafael Molano, quién con la intención de obtener recursos adicionales a los de su empleo, inició la venta de ponqués con la receta tradicional de su mamá, inicialmente a sus compañeros de trabajo y posteriormente en tiendas cercanas, en éstas últimas, en un principio no tuvo mucho éxito, pues los ponqués en ésa época sólo se vendían para cumpleaños y fechas especiales, sin embargo, se cuenta, que en ése momento el señor Molano pidió a uno de los tenderos un cuchillo y tajó el

3. La innovación parte de la utilización de conocimiento existente, la creación o generación de nuevo conocimiento no implica necesariamente que haya innovación. ponqué en porciones para así poder vender de manera individual y a un precio más asequible. La idea fue un éxito y a la semana la red de tiendas se había extendido considerablemente (Bermúdez, 2014), dando origen así a la que es hoy una de las empresas más representativas del país.

De acuerdo con el caso anterior, es válido afirmar que los elementos clave para que una innovación se presente, y que a su vez permita introducir el enfoque de alcance que ésta puede tener, son: la novedad (invento, adaptación, imitación o copia), las capacidades (compuestas por el conocimiento, tácito y explicito, y las habilidades) y el contexto que la aproveche (mercado), como se muestra en la figura 1; sin la presencia de estos elementos se puede decir que no es posible lograr conseguir una innovación, de manera tal que si algo carece de novedad, aunque se cuente con las capacidades para utilizarlo o aprovecharlo en un contexto específico no se da la innovación, por otro lado, si éste mismo elemento representa una novedad en otro contexto, donde además se cuente con (o haya disposición de crear) las capacidades para su introducción, si puede considerarse como innovación.

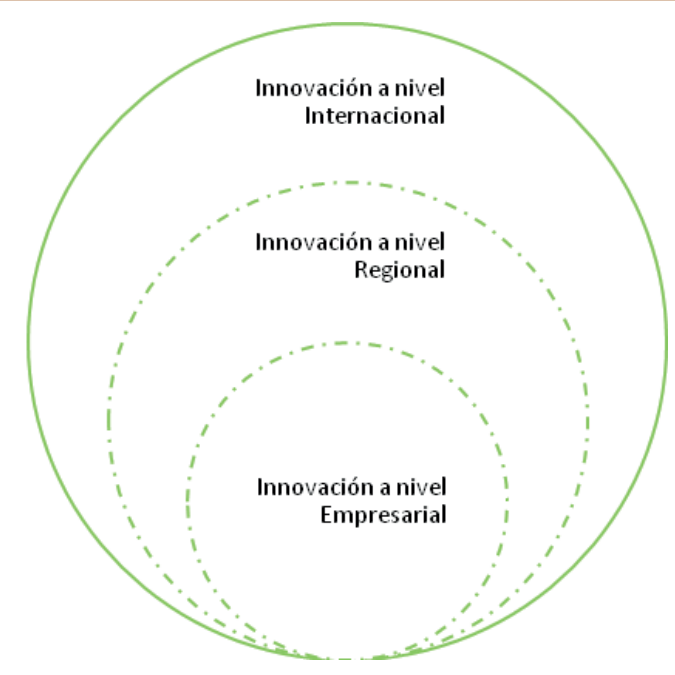

Figura 2. Alcance de la innovación. Fuente. Elaboración propia. 
Vista entonces la innovación como la aplicación exitosa de algo novedoso en un contexto específico, donde se aprovechen las capacidades y potencialidades, es válido afirmar que existen diferentes niveles o alcances de ésta, siendo posible que se presente al interior de una empresa (o en un gremio de empresas), en un área geográfica (provincia, país o región), o a nivel internacional, con la posibilidad de presentarse simultáneamente en varios de ellos ${ }^{4}$, como se muestra en la figura 2. El ejemplo mencionado de la empresa Ramo S.A., representa claramente una innovación -en términos de la Organización para la Cooperación y el Desarrollo Económicos (OCDE)- de método de comercialización la cual a su vez es de alcance local, pues en esa época los productos de panadería de venta en tienda no eran algo nuevo en otros contextos ${ }^{5}$.

De ésta manera, es el contexto el que tiene relación directa con el alcance esperado de la innovación, pues algo que es novedoso para una empresa o en un contexto dado puede no serlo para otra; de igual modo que para un sector industrial, área geográfica

4. De manera análoga a la concepción de capacidades tecnológicas propuesta por Lugones, Gutti E Le Clech (2007), también se puede hablar de innovación de alcance microeconómico (a nivel de empresas), mesoeconómico (sectorial) o macroeconómico (a nivel país e internacional), sin embargo en éste trabajo se hará referencia a los niveles presentados en la figura 2.

5. El caso de un producto de panadería tajado no nace a partir de éste momento, claramente desde antes era posible tajar un pan o un ponqué, sin embargo su comercialización en tajadas no se había dado en Colombia hasta esa fecha. Otro elemento que ratifica el carácter de innovación de alcance local es que en el año 1945 nace en México la empresa Bimbo, cuyo principal producto es el pan blanco tajado. o a nivel internacional. Un ejemplo de esto puede ser un hipotético caso de una empresa Latinoamericana productora de arroz, de la cual sus directivos asisten a un congreso y feria exposición realizados en Asia sobre avances tecnológicos entorno a éste alimento, allí se encuentran con una semilla que requiere una menor cantidad de agua durante el cultivo. Hasta aquí el caso es de una innovación internacional (donde podría decirse que se generó un conocimiento nuevo a nivel mundial), con gran interés los empresarios contactan con el proveedor de la semilla para establecer los términos para el uso de ésta (condiciones para la transferencia de conocimiento y/o tecnología que permita adquirir las capacidades), con lo cual se puede lograr una innovación en el mercado local (región o área geográfica), que a su vez también puede considerarse innovación entre las empresas del sector (empresarial).

Esto mismo es descrito en el Manual de Bogotá (Rycit, OEA, Colciencias \& Ocyt, 2001), desde el punto de vista del cambio tecnológico continuo, dado por la difusión de tecnología, que permite la adaptación de ésta en nuevos contextos, en los cuales al ser aplicadas permitirán alcanzar mayores niveles de eficiencia operativa; se parte de la idea de que en los Países Desarrollados (PD) se centra en mayor medida la actividad de innovación tecnológica pura, es decir innovación de alcance internacional, por su parte, en los PED se dan principalmente actividades de difusión y transferencia de las tecnologías ya creadas, lo que lleva a que especialmente se puedan presentar innovaciones a nivel regional o de empresa; a su vez en los PED se puede decir que se cuenta especialmente con "capacidades de producción”. 


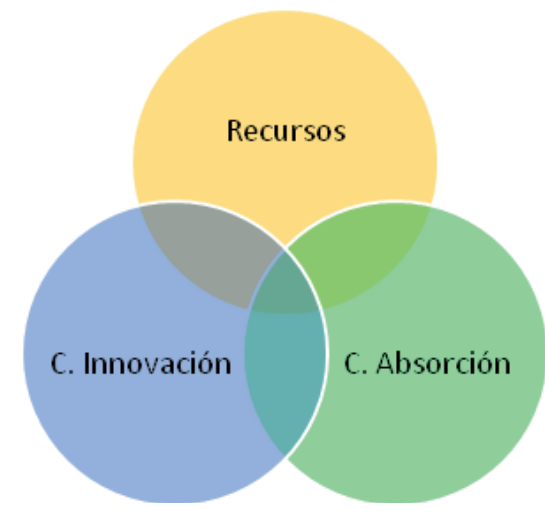

Figura 3. Elementos de la capacidad tecnológica.

Fuente. Elaboración propia.

Así es como el contexto define el alcance y la calidad de innovación en términos del éxito en la utilización de algo novedoso, por lo que claramente es necesario contar con las capacidades suficientes para la articulación de la novedad. A éste respecto se puede establecer que los PED deben enfocar su esfuerzo en consolidar sus capacidades, a fin de poder absorber, adaptar, mejorar y generar conocimiento y nuevas tecnologías (Lugones, Gutti, \& Le Clech, 2007), representadas en recursos disponibles -suficientes y adecuados-, así como de capacidades de absorción -reconocer el valor del conocimiento nuevo y externo, asimilarlo y aplicarlo- y de innovación -llegar al mercado o lograr el éxito con la inserción de la novedad-; a éste conjunto de elementos se puede denominar de modo genérico como "Capacidades Tecnológicas" (Gutti, 2015; RYcit et al., 2001), en la figura 3 se representan los elementos que componen dicha capacidad.

Así entonces, estableciendo que la innovación claramente puede presentarse a diferentes niveles, y que para lograrla se debe contar con un conjunto de capacidades, para los PED es importante distinguir el alcance de la innovación para así identificar las áreas en las que se debe priorizar el fortalecimiento de la capacidad tecnológica.

\section{REFERENCIAS}

Bermúdez, E. (2014, agosto 23). Rafael Molano: el cerebro detrás de los ponqués Ramo. El Tiempo. Recuperado en: http://www.eltiempo.com/estilo-de-vida/ gente/historia-de-rafael-molano-fundador-de-ponques-ramo/14427959

Gutti, P. (2015). Clase 6 - Convergencia: el rol de las "capacidades." Buenos Aires.

Lugones, G. E., Gutti, P. \& Le Clech, N. (2007). Indicadores de capacidades tecnológicas en América Latina (No. 89).México:Naciones Unidas.

OEdc. (2005). Manual de Oslo: guía para la recogida e interpretación de datos sobre innovación. Recuperado en: http://www.itq. edu.mx/convocatorias/manualdeoslo.pdf

Real Academia Española. (2014). Diccionario de la lengua española. Recuperado en::http://lema.rae.es/drae/?val=fenomeno

Rycit, OEA, Colciencias \& Ocyt. (2001). Manual de Bogotá: Normalización de Indicadores de Innovación Tecnológica en América Latina y el Caribe. Recuperado en: http://www.ricyt.org/manuales/doc_ view/5-manual-de-bogota 